\title{
Prediction of Pretreatment I8F-FDG-PET/CT Parameters on the Outcome of First-Line Therapy in Patients with Metastatic Breast Cancer
}

\author{
Yi Li, ${ }^{1} *$ Cheng Liu, ${ }^{2-6, *}$ \\ Bibo Wang, ${ }^{7, *}$ Xichun Hu, \\ Chengcheng Gong,' \\ Yannan Zhao,' Yizhao Xie,' \\ Yingjian Zhang, ${ }^{2-6}$ \\ Shaoli Song, ${ }^{2-6}$ \\ Zhongyi Yang, ${ }^{2-6}$ \\ Biyun Wang'
}

'Department of Medical Oncology, Fudan University Shanghai Cancer Center,

Department of Oncology, Shanghai Medical College, Fudan University, Shanghai, People's Republic of China; ${ }^{2}$ Department of Nuclear Medicine, Fudan University Shanghai Cancer Center, Shanghai, People's Republic of China; ${ }^{3}$ Department of Oncology, Shanghai Medical College, Fudan University, Shanghai, People's Republic of China; ${ }^{4}$ Center for Biomedical Imaging, Fudan University, Shanghai, People's Republic of China; ${ }^{5}$ Shanghai Engineering Research Center of Molecular Imaging Probes, Shanghai, People's Republic of China; ${ }^{6}$ Department of Nuclear Medicine, Shanghai Proton and Heavy lon Center, Shanghai, People's Republic of China; ${ }^{7}$ Orthopaedic Department, Shanghai Ruijin Hospital, Shanghai Jiaotong University Medicine School, Shanghai, People's Republic of China

*These authors contributed equally to this work

Correspondence: Biyun Wang Department of Medical Oncology, Fudan University Shanghai Cancer Center, No. 270 Dong'an Road, Shanghai, 200032, People's Republic of China

Email wangbiyun0107@hotmail.com

Zhongyi Yang

Department of Nuclear Medicine, Fudan University Shanghai Cancer Center, \#270 Dongan Road, Shanghai, 200032, People's Republic of China Email yangzhongyi2I@I63.com
Objective: 18F-fluorodeoxyglucose positron emission tomography/computed tomography (18F-FDG-PET/CT) can provide prognostic information, especially $18 \mathrm{~F}-\mathrm{FDG}$ uptake has been proven to be a predictor for the prognosis of various tumors. Nevertheless, the prognosis of other PET parameters in the metastatic setting remains unclear. This study was aimed at investigating pretreatment parameters based on $18 \mathrm{~F}-\mathrm{FDG}-\mathrm{PET} / \mathrm{CT}$ so as to estimate the progression-free survival (PFS) of metastatic breast cancer (MBC) patients receiving first-line treatment.

Methods: MBC patients who underwent a whole-body $18 \mathrm{~F}-\mathrm{FDG}-\mathrm{PET} / \mathrm{CT}$ prior to first-line therapy were enrolled. The heterogeneity parameter of PET/CT was analyzed, including heterogeneity index (HI) and general parameters (metabolic tumor volume (MTV), total lesion glycolysis (TLG), maximum standardized uptake value (SUVmax) and mean SUV (SUVmean). PFS was used to evaluate the treatment outcome. Kaplan-Meier method was adopted to carry out survival analysis and $\log$ rank test was conducted to make a comparison.

Results: A total of $177 \mathrm{MBC}$ patients were selected, in which 68 were in De novo stage IV. Thirty patients were human epidermal growth factor receptor 2 (HER2)-positive, 60 patients were triple-negative, and 87 patients were hormone receptor (HR)-positive and HER2negative. In the whole population, patients with high baseline SUVmax, SUVmean, MTV, TLG or HI were associated with lower PFS $(P=0.028,0.005,0.017,0.026$ and 0.035 , respectively). Among the patients in De novo stage IV, those with high $\mathrm{HI}$ at baseline had significantly shorter PFS $(P=0.001)$. In HR+/HER2- and HER2+ subgroups, only baseline HI showed the predictive value of PFS ( $P=0.023$ and 0.049 , respectively). In the triple-negative subgroup, high baseline SUVmax, MTV or TLG showed the predictive value of worse PFS $(P=0.030,0.011$ and 0.023 , respectively).

Conclusion: Pretreatment 18F-FDG-PET/CT parameters show the predictive value of PFS in MBC patients receiving first-line treatment. However, predictive PET/CT parameters might be different in patients with different molecular subtypes and De novo stage IV.

Keywords: metastatic breast cancer, FDG-PET/CT, parameter, prognosis, molecular subtypes

\section{Introduction}

As the most prevalent malignant tumor, breast cancer (BC) was the major reason for the death of women worldwide. ${ }^{1}$ About $6 \%$ of $\mathrm{BC}$ patients have metastatic disease when they are diagnosed, and around $30 \%$ of early-stage patients would 
finally develop metastatic breast cancer (MBC). ${ }^{2}$ The fiveyear relative survival rate of women diagnosed with earlystage disease is significantly higher than that of women diagnosed with distant metastatic disease at presentation. ${ }^{3}$ In spite of the great improvements achieved in the past 10 years, the median five-year survival rate of MBC is only $33.8 \%{ }^{4}$ The triple-negative and human epidermal growth factor receptor 2 (HER2)-positive subgroups of BC used to be considered as aggressive phenotypes with poor prognosis compared with the luminal subgroup. ${ }^{5}$ However, the development of anti-HER2 targeted therapies has significantly improved the prognosis of HER $2+$ BC. ${ }^{4}$

Tumor volume based on morphological or functional imaging could be used to identify patients with a worse prognosis. ${ }^{6}$ Indeed, 18F-fluorodeoxyglucose (18F-FDG) positron emission tomography/computed tomography (PET/CT) parameters, especially maximum standard uptake value (SUVmax), are demonstrated to be correlated with the clinical outcome of BC. ${ }^{7-9}$ Besides, studies have shown the predictive value of heterogeneity on the basis of $18 \mathrm{~F}-\mathrm{FDG}-\mathrm{PET} / \mathrm{CT}$ in various primary tumors like early breast cancer (EBC). ${ }^{10,11}$ However, few studies have reported other imaging classifiers, such as mean SUV (SUVmean), heterogeneity index (HI), total lesion glycolysis (TLG), metabolic tumor volume (MTV) or their correlation with the prognosis of MBC. The research results of primary lesions might be inapplicable to metastatic disease since metastases could show completely different behaviors in tumor metabolism and biology compared with primary tumors or between each other. ${ }^{12,13}$

This study aimed to examine 18F-FDG pretreatment imaging parameters (including SUVmax, SUVmean, TLG, MTV and HI) as predictors for the first-line progressionfree survival (PFS) of MBC patients with different molecular subtypes and De novo stage IV.

\section{Methods}

\section{Patients}

177 MBC patients undergoing an FDG-PET/CT at FUSCC were enrolled. Inclusion criteria: MBC patients who had no less than one measurable metastatic disease with the abnormal uptake of FDG and without systemic or local therapy for metastatic disease. Exclusion criteria: Patients who had diabetes and uncontrolled brain metastases, and were pregnant or lactating, diagnosed with a second primary malignancy and actively or uncontrollably infected. For patients with many metastatic sites, the lesion with the highest SUVmax was utilized. Medical records were used to collect the data on the characteristics of patients and their treatment outcomes. Hormone receptor (HR)positive refers to the tumor tissue expressing estrogen or progesterone receptors in no less than $10 \%$ of cells based on local lab parameters. Local assessment defines HER 2 + to be positive for immunohistochemical 3+ and/or fluorescent in situ hybridization-positive. Tumor tissue includes primary breast cancer (PBC) or metastatic tumor tissue. Patients were required to have follow-up in FUSCC every two months.

\section{$\mathrm{PET} / \mathrm{CT}$}

The Explora FDG4 module of the center was used to automatically produce $18 \mathrm{~F}-\mathrm{FDG}$ achieving more than $95 \%$ of radiochemical purity using a cyclotron (Siemens CTI RDS Eclips ST, Knoxville, Tennessee, the US). Before 18F-FDG PET, patients were asked to fast for at least six hours to keep their blood glucose level under 10 $\mathrm{mmol} / \mathrm{L}$. In addition, all of them were injected intravenously with $7.4 \mathrm{MBq} / \mathrm{kg}$ of $18 \mathrm{~F}-\mathrm{FDG}$. After that, patients were asked to rest in a quiet and dark room for 60 minutes, and then the images were acquired through a biography 16HR PET/CT scanner (Knoxville, Tennessee, the US). $\mathrm{PET} / \mathrm{CT}$ was performed by scanning from the middle of the skull to the supine position at mid-thigh. The process of acquiring PET/CT parameters was as follows: First, a whole-body CT was performed $(120 \mathrm{kV}, 80 \sim 250 \mathrm{~mA}$, pitch 3.6, rotation time was $0.5 \mathrm{~s}$ ). Then, PET covering the same lateral view was conducted. The collection time for each workbench position was 2 to 3 minutes. CT data were applied to correct attenuation, and the dataset of PET images was iteratively reconstructed by using Ordered Subset Expectation Maximization (OSEM), an ordered subset. Fused PET/CT images were displayed on a Syngo multimodality computer platform (Siemens, Knoxville, Tennessee, the US) for analysis.

\section{Image Analysis}

Two board-certified seasoned nuclear medicine physicians major in 18F-FDG-PET/CT images made the evaluation independently. Any disagreement would be solved with the consensus between the two reviewers on the final reading of statistical analysis. All hypermetabolic metastatic lesions were selected for analysis without the consideration of hypermetabolic foci assessed by 
inflammation or physiological activities. SUV normalized to body weight was applied to carry out a semiquantitative analysis of tumor metabolic activities. All parameters were evaluated in a $3 \mathrm{D}$ volume. To determine the contour edges around the lesion, $\mathrm{SUV}>2.5$ was used. A single region of interest (ROI) was placed around every lesion on all the consecutive slices containing lesions to record the SUVmax and SUVmean of each metastatic lesion, document and fuse axial PET/CT images. Then, MTV was extracted from software in an automatic way according to the SUV threshold of 2.5. The formula that TLG $=$ SUVmean $\times$ MTV was used to calculate TLG. HI in tumors was quantitatively measured by dividing SUVmax by SUVmin. ${ }^{14,15}$

\section{Statistical Analysis}

Each distribution was represented as the median (range) or amount (percentage) of quantitative or categorical data, respectively. The median was chosen as the cut-off value of image parameters. The treatment effect was assessed using PFS, which meant the date from firstline therapy to disease progression was recorded for the first time, death or final follow-up. Disease progression was obtained based on version 1.1 of Response Evaluation Criteria in Solid Tumors (RECIST). KaplanMeier method was adopted to estimate the median PFS and the corresponding $P$ values and 95\% confidence interval (CI). The relationship between baseline characteristics, values of image parameters and PFS was examined by a Log rank test. Univariate analysis was estimated using Cox proportional hazard models that were denoted by hazard ratios (HRs) with corresponding $P$ values and 95\% CI. The effects of variables were expressed as HRs with 95\% CI and P values. Every analysis was double-sided. It was considered that $P$ value below 0.05 had no statistical significance. The software SPSS 22.0 (IBM Corporation, Armonk, New York, the US) was used for data analysis.

\section{Results}

\section{Characteristics and Treatment Outcome of Patients}

$177 \mathrm{MBC}$ patients undergoing PET/CT before first-line therapy were enrolled, with the median age of 53 (ranging from 29 to 74). And their baseline characteristics are shown in Table 1. Of the 177 patients, 68
Table I Patients' Characteristics at Baseline

\begin{tabular}{|c|c|}
\hline Characteristics & MBC, $n=177, n$ (\%) \\
\hline Age, years & \\
\hline Median (range) & $53(29-74)$ \\
\hline Menopausal status & \\
\hline Postmenopausal & $105(59.3)$ \\
\hline Premenopausal & $72(40.7)$ \\
\hline De novo & $68(38.4)$ \\
\hline Disease-free interval, months & \\
\hline$<24$ & $49(27.7)$ \\
\hline$\geq 24$ & $60(33.9)$ \\
\hline Subgroups & \\
\hline HR+/HER2- & $87(58.2)$ \\
\hline HER 2+ & $30(16.9)$ \\
\hline Triple-negative & $60(33.9)$ \\
\hline Number of metastatic sites & \\
\hline I & $5 I(28.9)$ \\
\hline 2 & $62(35.0)$ \\
\hline$\geq 3$ & $64(36.1)$ \\
\hline Metastatic sites & \\
\hline Liver & $33(18.6)$ \\
\hline Lung & 67 (37.9) \\
\hline Bone & $94(53.1)$ \\
\hline Visceral disease & $96(54.2)$ \\
\hline First-line treatment & \\
\hline Endocrine therapy & $44(24.9)$ \\
\hline Anti-HER2 therapy & $29(16.4)$ \\
\hline Chemotherapy & $104(58.7)$ \\
\hline
\end{tabular}

Abbreviations: HR+, hormone receptor positive; HER2-, human epidermal growth factor receptor 2 negative.

(38.4\%) were in De novo stage IV without receiving any prior treatment for BC. The observed subtypes were as follows: HR+/HER2- of 87 (49.2\%), HER2overexpressing of $30(16.9 \%)$ and triple-negative of 60 (33.9\%). In HR+/HER2- group, 40 patients received endocrine therapy without the combination with targeted agents as their first-line treatment, while 47 patients were treated with chemotherapy. All HER2+ patients received first-line anti-HER2 treatment and all triple-negative patients received first-line chemotherapy. The common metastatic sites of FDG-PET/CT were bone of 94 (53.1\%), lung of 67 (37.9\%) and liver of $33(18.6 \%)$, with 64 patients presenting more than three metastatic sites. Visceral involvement was present in more than half of the patients (54.2\%). 44 
Table 2 Analysis of Risk Factors Associated with PFS in the Whole Population

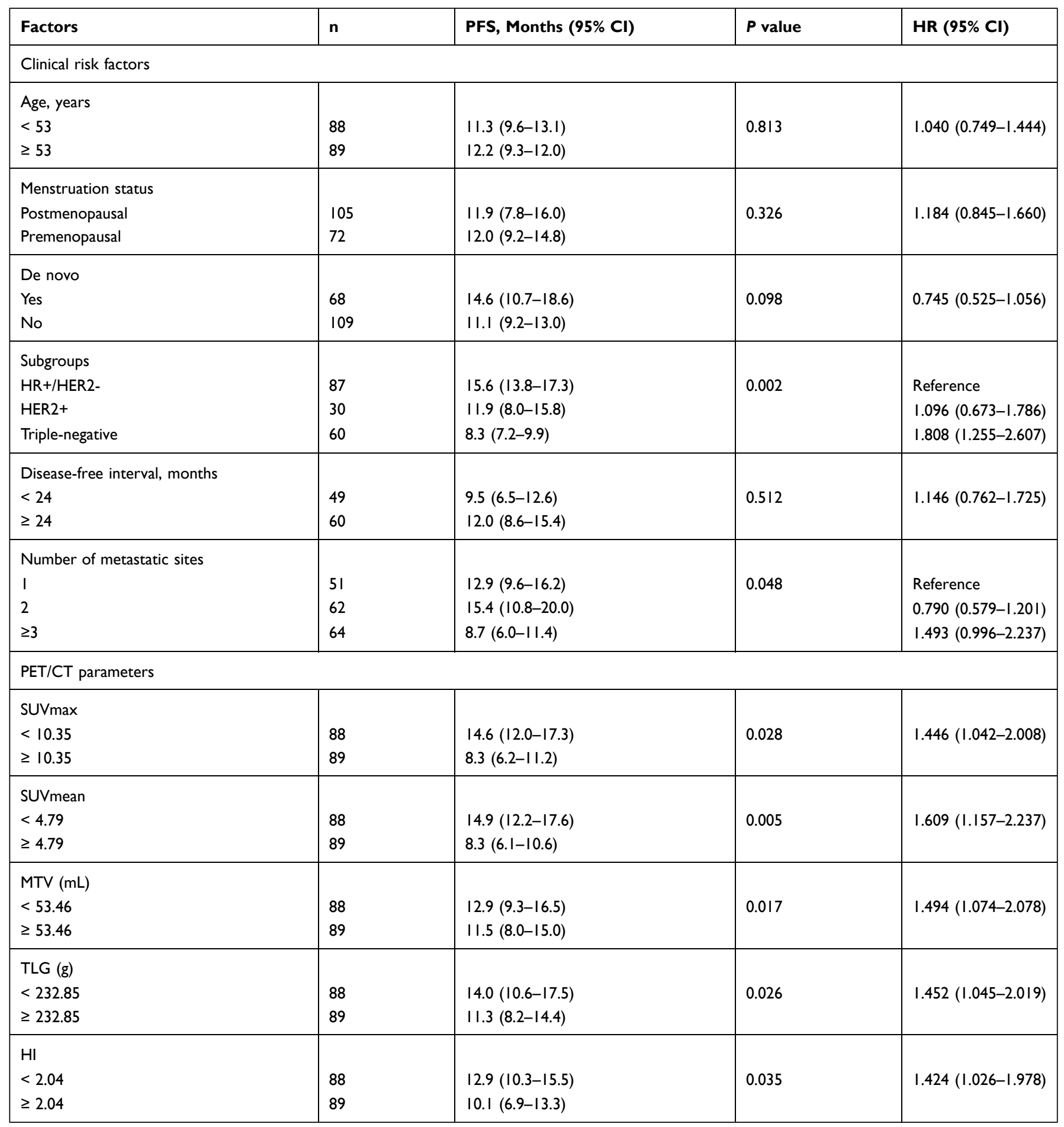

Abbreviations: PFS, progression-free survival; $\mathrm{HR}$, hazard ratio; $\mathrm{Cl}$, confidence interval; $\mathrm{HR+}$, hormone receptor positive; HER2-, human epidermal growth factor receptor 2 negative; PET/CT, positron emission tomography/computed tomography; SUVmax, maximum standard uptake value; SUVmean, mean standard uptake value; MTV, metabolic volume measurements; TLG, total lesion glycolysis; HI, heterogeneity index.

patients underwent first-line endocrine therapy, antiHER2 therapy and chemotherapy, respectively. 1,387 metastatic tumors were measured and analyzed.
After receiving a median follow-up of 24.0 months (ranging from 3.1 to 102.0 months), 144 (81.4\%) patients experienced disease progression and 41 
(23.2\%) patients died, leading to the median PFS of 12.0 months (95\% CI: 10.2-13.7) (Supplementary Figure 1). Median overall survival (OS) was not reached in the last follow-up.

\section{Baseline Characteristics and PFS}

Molecular subtypes were statistically significant and related to median PFS. Patients with HR+/HER2- presented the best median PFS of 15.5 months, while patients with triple-negative presented the worst median PFS of 8.6 months $(P=0.002)$. Patients with fewer metastatic sites had significantly longer PFS than those with $\geq 3$ metastatic sites $(P=0.048)$. A correlation without statistical significance was observed between De novo stage IV and PFS (Table 2). The other baseline characteristics had no significant association with PFS.

\section{Association Between PFS and Pretreatment PET Parameters}

The median values of SUVmax, SUVmean, MTV, TLG and HI determined their cut-off values, which were $10.35,4.79,53.46 \mathrm{~mL}, 232.85 \mathrm{~g}$ and 2.04 , respectively
(Table 2). Univariate analysis was used to evaluate the prognostic value of pretreatment parameters. High baseline SUVmax, SUVmean, MTV, TLG and HI were all significantly correlated with worse PFS ( $P=0.028,0.005,0.017,0.026$, and 0.035 , respectively, Figure 1 and Table 2). In De novo stage IV patients, there was no significant association between SUVmax, SUVmean, MTV or TLG and PFS ( $P=0.177,0.065$, 0.343 and 0.214 , respectively, as shown in Table 3 ), and only patients with high baseline HI showed obviously shorter PFS compared with those with low baseline HI (HR, 2.821; 95\% CI, 1.544-5.154; $P=0.001$, Figure 2).

PET-derived parameters might vary widely in different molecular subtypes. Therefore, separate analyses were performed for each molecular subtype (Table 4). Only baseline HI showed the predictive value of PFS in HR+/HER2- subgroup, in which patients with high baseline HI had obviously worse PFS than those with low baseline HI (HR, 1.711; 95\% CI, 1.070-2.734; $P=0.025$, Figure 3). In the HER2+ subgroup, only patients with high baseline HI showed obvious shorter median PFS
A

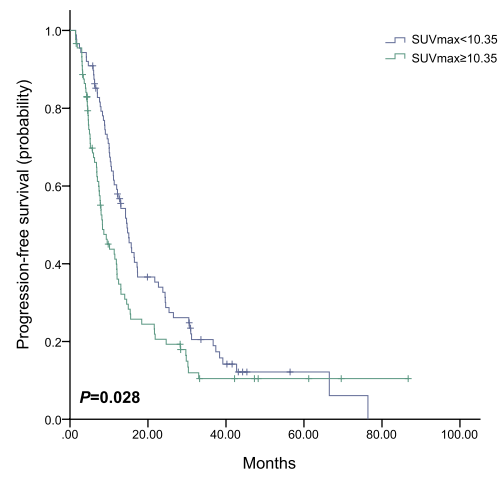

D

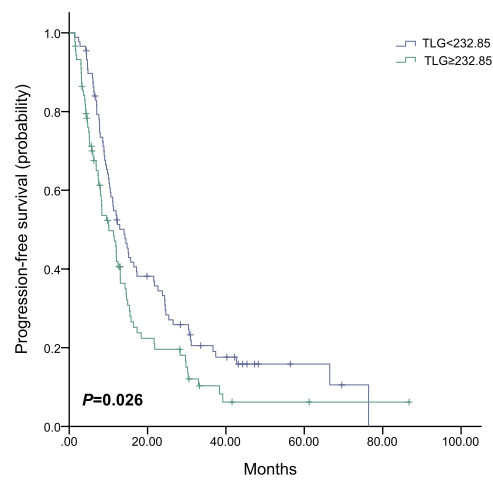

B

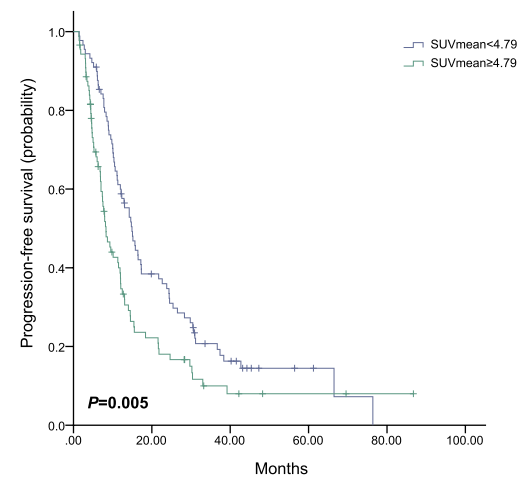

E

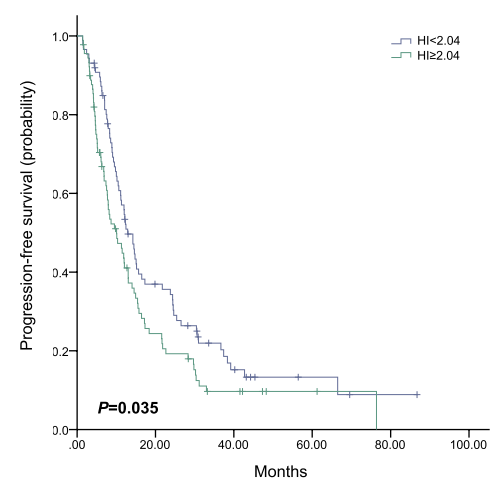

C

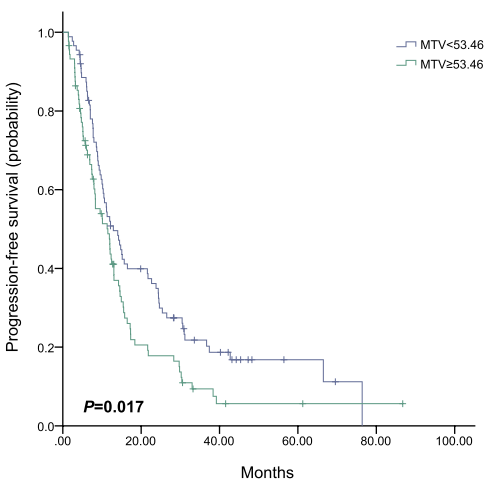

Figure I Kaplan-Meier curve of progression-free survival for all patients stratified by SUVmax (A), SUVmean (B), MTV (C), TLG (D) and HI (E). Abbreviations: SUVmax, maximum standardized uptake value; SUVmean, mean SUV; MTV, metabolic tumor volume; TLG, total lesion glycolysis; HI, heterogeneity index. 
Table 3 Analysis of PET Parameters Associated with PFS in Patients with De novo Stage IV

\begin{tabular}{|c|c|c|c|c|}
\hline \multicolumn{5}{|c|}{ In Patients with De novo Stage IV } \\
\hline Factors & $\mathbf{n}$ & $\begin{array}{l}\text { PFS, Months } \\
(95 \% \mathrm{CI})\end{array}$ & $P$ value & HR (95\% Cl) \\
\hline $\begin{array}{l}\text { SUVmax } \\
<12.79 \\
\geq 12.79\end{array}$ & $\begin{array}{l}34 \\
34\end{array}$ & $\begin{array}{l}17.2(4.0-30.4) \\
8.2(3.9-12.4)\end{array}$ & 0.177 & $\mathrm{I} .475(0.839-2.593)$ \\
\hline $\begin{array}{l}\text { SUVmean } \\
<5.28 \\
\geq 5.28\end{array}$ & $\begin{array}{l}34 \\
34\end{array}$ & $\begin{array}{l}22.7(9.5-35.9) \\
10.7(6.3-14.8)\end{array}$ & 0.110 & $1.590(0.900-2.809)$ \\
\hline $\begin{array}{l}\operatorname{MTV}(\mathrm{mL}) \\
<109.93 \\
\geq 109.93\end{array}$ & $\begin{array}{l}34 \\
34\end{array}$ & $\begin{array}{l}\text { I8.4 (5.6-3|.2) } \\
|3 .|(9.0-|7 .|)\end{array}$ & 0.343 & $1.320(0.744-2.340)$ \\
\hline $\begin{array}{l}\text { TLG }(g) \\
<571.56 \\
\geq 571.56\end{array}$ & $\begin{array}{l}34 \\
34\end{array}$ & $\begin{array}{l}\text { I8.4 (2.7-34.I) } \\
\text { I2.I (9.3-14.8) }\end{array}$ & 0.214 & $\mathrm{I} .44 \mathrm{I}(0.810-2.563)$ \\
\hline $\begin{array}{l}\mathrm{HI} \\
<2.67 \\
\geq 2.67\end{array}$ & $\begin{array}{l}34 \\
34\end{array}$ & $\begin{array}{l}25.4(4.6-46.2) \\
8.2(3.1-13.3)\end{array}$ & 0.001 & $2.821(1.544-5.154)$ \\
\hline
\end{tabular}

Abbreviations: PET/CT, positron emission tomography/computed tomography; PFS, progression- free survival; $\mathrm{HR}$, hazard ratio; $\mathrm{Cl}$, confidence interval; SUVmax, maximum standard uptake value; SUVmean, mean standard uptake value; MTV, metabolic volume measurements; TLG, total lesion glycolysis; HI, heterogeneity index.

(HR, 2.555; 95\% CI, 1.006-6.493; $P=0.049$, Figure 4). However, patients with high baseline SUVmax, MTV or TLG had worse prognosis in the triple-negative subgroup (HR,1.862; 95\% CI, 1.061-3.267; $P=0.030$; HR, 2.053; 95\% CI, 1.177-3.579; $P=0.011$; HR, 1.901; 95\% CI, 1.091-3.311; $P=0.023$, respectively, Figure 5). Images of the representative tumors of $\mathrm{HR}+/ \mathrm{HER} 2-$ are shown in Figure 6.

\section{Discussion}

Previous studies reported the prognostic value of PETderived parameters in $\mathrm{BC}$, but most of them focused on EBC and PET parameters were limited to only SUVmax. ${ }^{7-9}$ No research was conducted on the prognostic value of different PET parameters in $\mathrm{MBC}$ patients, especially those with De novo stage IV or different molecular subtypes. To our knowledge, this is the first relatively large-scale and retrospective report on the predictive value of PET parameters in metastatic treatment naïve $\mathrm{MBC}$ patients stratified by De novo stage IV and molecular subtypes. Aimed at assessing the predictive value of baseline PET-derived parameters in patients with MBC before first-line treatment, this study performed a retrospective analysis on 177 patients who underwent an $18 \mathrm{~F}-\mathrm{FDG}$ PET/CT scan prior to the treatment.

In this study, MBC patients with high baseline SUVmax, SUVmean, TLG, MTV and HI were all significantly correlated with worse PFS. Studies had reported the predictive value of SUVmax in EBC, but its role in $\mathrm{MBC}$ was not fully understood. The predictive value of SUVmax in unselected MBC patients was demonstrated by previous research. However, the prognostic value of other PET parameters was not explored. ${ }^{16}$ Morris et $\mathrm{al}^{9}$ reported that the increase in SUVmax in bone was associated with lower survival in newly diagnosed $\mathrm{MBC}$ patients, and the similar effects were observed in other metastatic sites but without showing statistical significance.

SUVmax and SUVmean could be limited by the volume incorporating non-metabolically active disease. ${ }^{17}$ To overcome this limitation, other measures have been proposed, such as TLG and MTV. ${ }^{18}$ Calculated by determining all voxels showing FDG uptake above a certain percentage of SUVmax, MTV could be multiplied by the mean SUV from that lesion to obtain a parameter called total lesion glycolysis (TLG). ${ }^{18}$ Studies on a variety of tumors including BC showed that TLG or MTV was significantly correlated with survival, and their prognostic value was often higher than SUV measurements. ${ }^{19-21}$ Ulaner et al $^{19}$ demonstrated that TLG and MTV had prognostic strength in newly diagnosed MBC patients when stratified by the metastasis sites, but they failed to analyze the prognostic correlation between TLG, MTV and the total population. Our study assessed the predictive value of TLG and MTV in MBC patients, and found that patients with high baseline TLG or MTV showed a worse median PFS than those with low TLG or MTV.

Previous studies showed that the intratumor heterogeneity of baseline PET had a better predictive value for treatment response and outcomes in a variety of cancers, such as nasopharyngeal carcinoma, ${ }^{22}$ lung cancer $^{23}$ and EBC. ${ }^{10}$ MBC usually shows a high degree of heterogeneity in biological and metabolic behaviors. Thus, heterogeneity may serve as a prognostic indicator of treatment failure. We first explored the importance of $\mathrm{HI}$ derived from PET in unselected $\mathrm{MBC}$ 


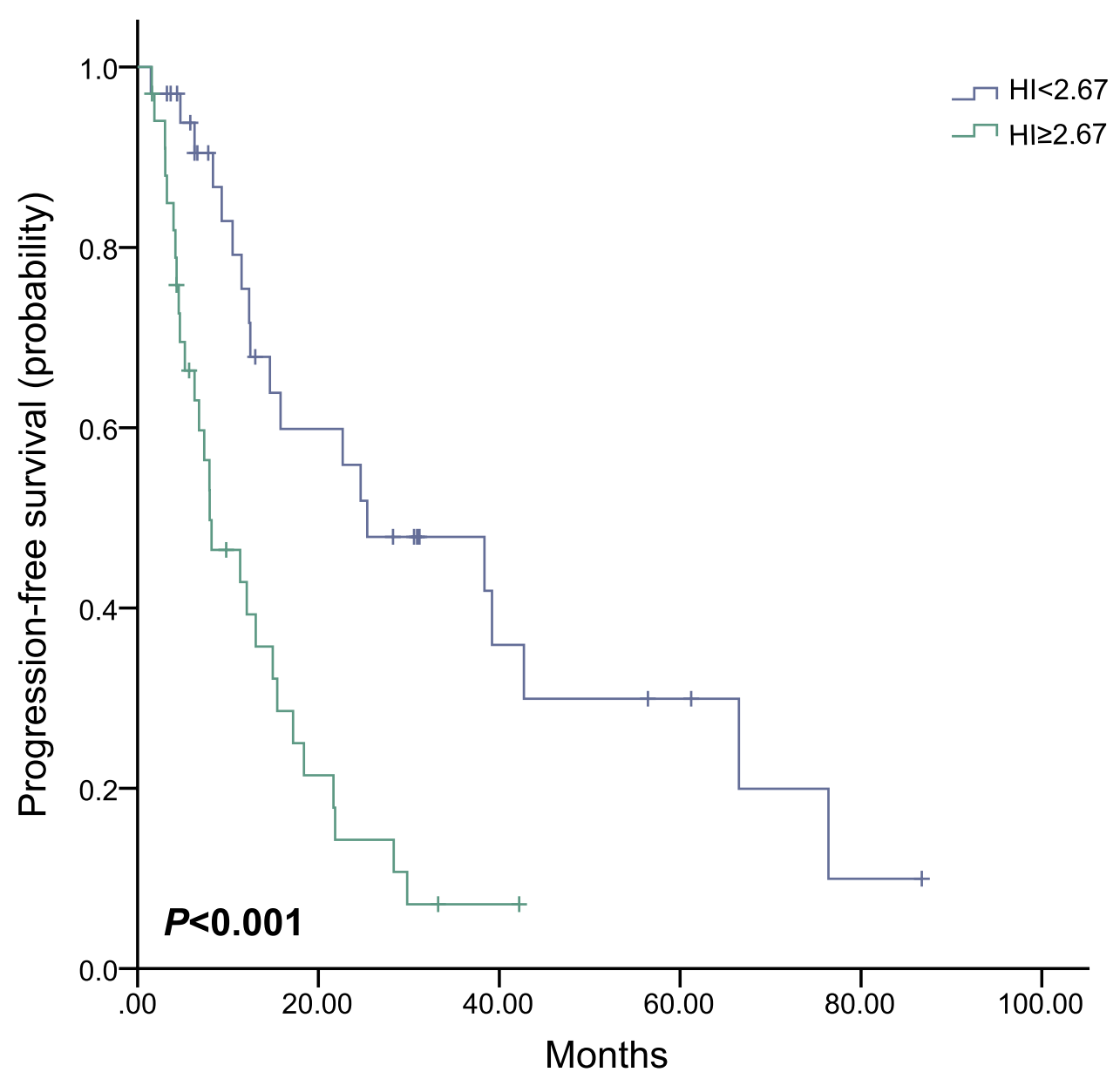

Figure 2 Kaplan-Meier curve of progression-free survival for patients with De novo stage IV stratified by $\mathrm{HI}$. Abbreviation: $\mathrm{HI}$, heterogeneity index.

patients without receiving first-line treatment. Then, intratumor heterogeneity was evaluated by $\mathrm{HI}$ and found to be significantly correlated with PFS. It is generally considered that a metastatic lesion extends from a cell to a colony, which may lead to the formation of intra-tumor heterogeneity that is probably more effective in reflecting metabolic status and treatment response, particularly in pretreatment tumors. Drug resistance often changes during treatment. ${ }^{24}$ Although responsive clones are eliminated, resistant clones are likely to reduce therapeutic success and cause treatment failure. ${ }^{25}$

Compared with general MBC, patients with De novo stage IV may contain more stem cells, which thus turn resistance to chemotherapy into a possible factor adversely affecting survival. This study investigated the prognostic value of pretreatment PET-derived parameters in De novo stage IV breast cancer patients. The results indicated that only baseline HI was significantly associated with PFS. The HI of these patients was supposed to be inherently higher, which was more likely to result in treatment failure and worse PFS.

Considering that the prognostic value of PET parameters in a mixed MBC population might be confused by the distribution of molecular subtypes in the study population, separate analyses were performed on different molecular subtypes. The predictive value of PFS was demonstrated using only baseline $\mathrm{HI}$ in $\mathrm{HR}$ +/HER2- and HER2+ subgroups, but shown by baseline SUVmax, MTV, TLG in the triple-negative subgroup. Previous studies showed that the baseline SUVmax of PET had the potential to predict the prognosis of $\mathrm{EBC}^{8}$ and $\mathrm{MBC}^{26}$ patients with luminal subtypes. Nevertheless, both studies included patients with 
Table 4 Analysis of PET Parameters Associated with PFS in Patients with Different Molecular Subtypes

\begin{tabular}{|c|c|c|c|c|}
\hline Factors & $\mathbf{n}$ & PFS, Months $(95 \% \mathrm{CI})$ & $P$ value & HR (95\% Cl) \\
\hline \multicolumn{5}{|c|}{ In HR+/HER2- patients } \\
\hline \multicolumn{5}{|l|}{ SUVmax } \\
\hline$<9.08$ & 43 & $16.4(13.7-19.0)$ & 0.293 & $1.282(0.806-2.038)$ \\
\hline$\geq 9.08$ & 44 & $14.9(10.9-18.9)$ & & \\
\hline \multicolumn{5}{|l|}{ SUVmean } \\
\hline$<4.56$ & 43 & $17.2(8.1-26.3)$ & 0.070 & $1.539(0.966-2.453$ \\
\hline$\geq 4.56$ & 44 & $14.5(10.6-18.4)$ & & \\
\hline \multicolumn{5}{|l|}{ MTV (mL) } \\
\hline$<53.46$ & 43 & $16.5(8.1-24.9)$ & 0.203 & $1.355(0.848-2.164)$ \\
\hline$\geq 53.46$ & 44 & $14.9(12.1-17.8)$ & & \\
\hline \multicolumn{5}{|l|}{ TLG (g) } \\
\hline$<244.83$ & 43 & $17.2(8.7-25.7)$ & 0.277 & $1.296(0.812-2.069)$ \\
\hline$\geq 244.83$ & 44 & $14.9(11.7-18.2)$ & & \\
\hline \multicolumn{5}{|l|}{$\mathrm{HI}$} \\
\hline$<2.24$ & 43 & $21.7(|2.0-3| .4)$ & 0.025 & 1.711 (1.070-2.734) \\
\hline$\geq 2.24$ & 44 & $13.0(7.2-18.9)$ & & \\
\hline \multicolumn{5}{|c|}{ In HER2+ patients } \\
\hline \multicolumn{5}{|l|}{ SUVmax } \\
\hline$<12.29$ & 15 & II.3 (4.7-I4.9) & 0.520 & $0.753(0.3 \mid 8-1.784)$ \\
\hline$\geq 12.29$ & 15 & $14.0(8.3-19.8)$ & & \\
\hline \multicolumn{5}{|l|}{ SUVmean } \\
\hline$<5.09$ & 15 & $17.3(6.4-28.3)$ & 0.410 & $1.439(0.606-3.419)$ \\
\hline$\geq 5.09$ & 15 & II.3 (9.3-13.3) & & \\
\hline \multicolumn{5}{|l|}{ MTV (mL) } \\
\hline$<69.42$ & 15 & $14.0(1.5-35.4)$ & 0.151 & $1.956(0.783-4.884)$ \\
\hline$\geq 69.42$ & 15 & $11.3(9.3-13.4)$ & & \\
\hline \multicolumn{5}{|l|}{ TLG(g) } \\
\hline$<353.85$ & 15 & $14.0(1.5-35.4)$ & 0.151 & $1.956(0.783-4.884)$ \\
\hline$\geq 353.85$ & 15 & $11.3(9.3-13.4)$ & & \\
\hline \multicolumn{5}{|l|}{$\mathrm{HI}$} \\
\hline$<2.52$ & 15 & $24.7(2.0-6 \mathrm{I} .3)$ & 0.049 & $2.555(1.006-6.493)$ \\
\hline$\geq 2.52$ & 15 & II.3 (6.7-16.0) & & \\
\hline \multicolumn{5}{|c|}{ In triple-negative patients } \\
\hline \multicolumn{5}{|l|}{ SUVmax } \\
\hline$<11.17$ & 30 & $10.2(8.0-12.4)$ & 0.030 & $1.862(1.06 \mathrm{I}-3.267)$ \\
\hline$\geq 11.17$ & 30 & $6.9(3.8-10.0)$ & & \\
\hline \multicolumn{5}{|l|}{ SUVmean } \\
\hline$<5.13$ & 30 & $10.1(8.0-12.1)$ & 0.201 & $1.434(0.825-2.494)$ \\
\hline$\geq 5.13$ & 30 & $7.0(4.7-9.3)$ & & \\
\hline \multicolumn{5}{|l|}{ MTV (mL) } \\
\hline$<45.64$ & 30 & $10.2(7.8-12.5)$ & 0.011 & $2.053(1.177-3.579)$ \\
\hline$\geq 45.64$ & 30 & $5.8(2.7-8.8)$ & & \\
\hline
\end{tabular}

(Continued) 
Table 4 (Continued).

\begin{tabular}{|l|l|l|l|l|}
\hline Factors & $\mathbf{n}$ & PFS, Months $(95 \% \mathbf{C I})$ & P value & HR (95\% CI) \\
\hline TLG (g) & & & & \\
$<232.85$ & 30 & $9.5(7.4-11.6)$ & 0.023 & $1.901(1.091-3.311)$ \\
$\geq 232.85$ & 30 & $6.9(3.3-10.5)$ & & \\
\hline HI & & & 0.126 & $1.540(0.886-2.675)$ \\
$<1.76$ & 30 & $9.3(8.2-10.4)$ & & \\
$\geq 1.76$ & 30 & $6.9(3.8-10.0)$ & & \\
\hline
\end{tabular}

Abbreviations: PET/CT, positron emission tomography/computed tomography; PFS, progression- free survival; HR, hazard ratio; Cl, confidence interval; HR+, hormone receptor positive; HER2, human epidermal growth factor receptor 2 negative; SUVmax, maximum standard uptake value; SUVmean, mean standard uptake value; MTV, metabolic volume measurements; TLG, total lesion glycolysis; HI, heterogeneity index.

Luminal A and B rather than only HR+/HER2-. Another study analyzed locally advanced breast cancer (LABC) patients, showing that high SUVs, TLG and MTV led to shorter PFS, while homogeneity was not predictive. $^{27}$ However, findings of primary tumors might not be applicable to metastatic disease due to the difference between $\mathrm{MBC}$ and $\mathrm{LABC}$ and in the measurement of heterogeneity in the two studies. This study also explored the prognostic value of PET parameters in HER2+ MBC patients. All HER2+ patients enrolled received first-line anti-HER2 treatment, excluding one without receiving chemotherapy only for economic reasons, which could minimize the impact of treatment on the heterogeneity of tumors and the prognosis of patients. However, two studies on metastatic triple-negative breast cancer (mTNBC) showed different results in the prognostic value of PET parameters. Marinelli et $\mathrm{al}^{28}$ found that baseline

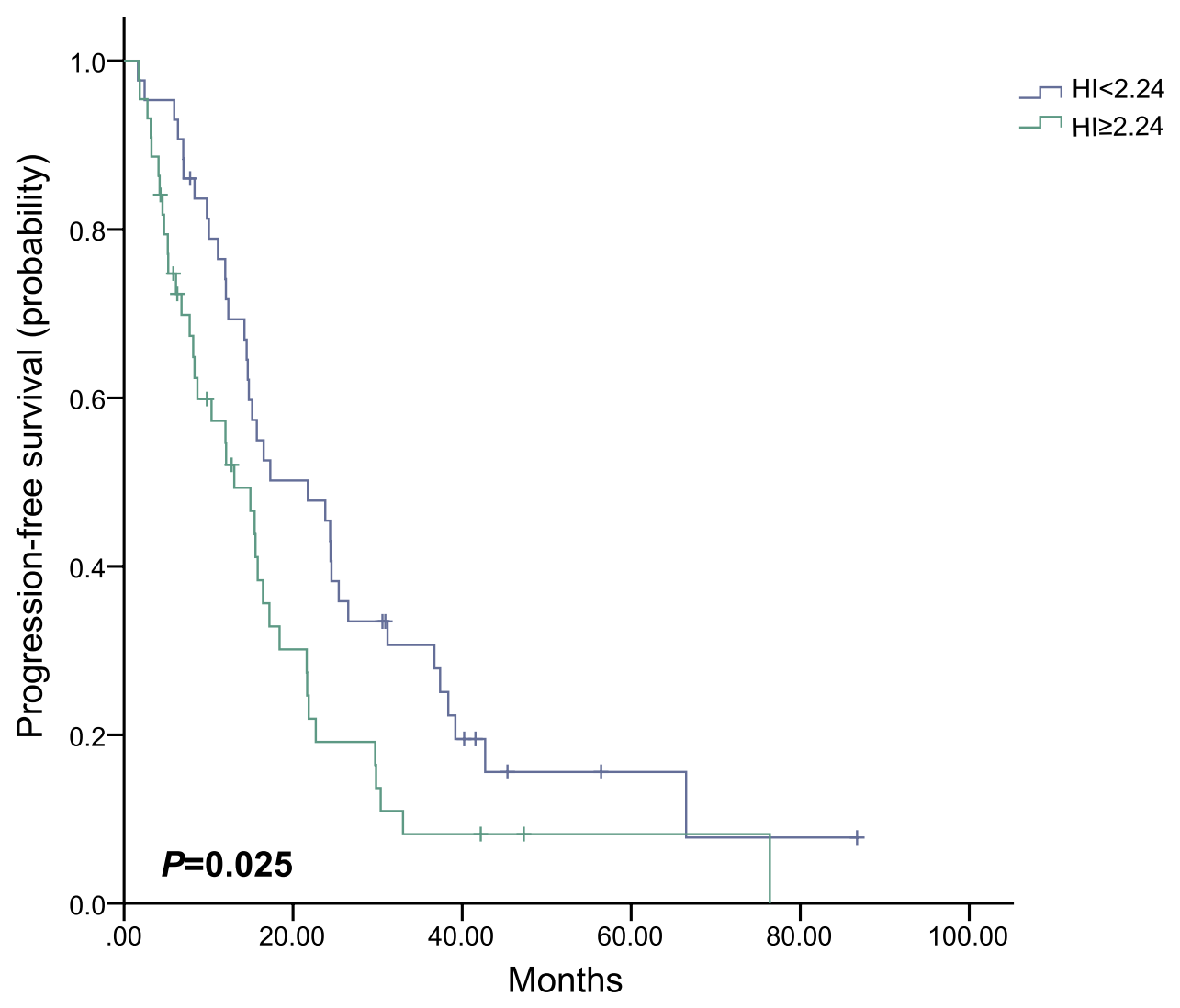

Figure 3 Kaplan-Meier curve of progression-free survival for HR+/HER2- patients stratified by HI.

Abbreviations: HR, hormone receptor, HER2, human epidermal growth factor receptor 2; HI, heterogeneity index. 


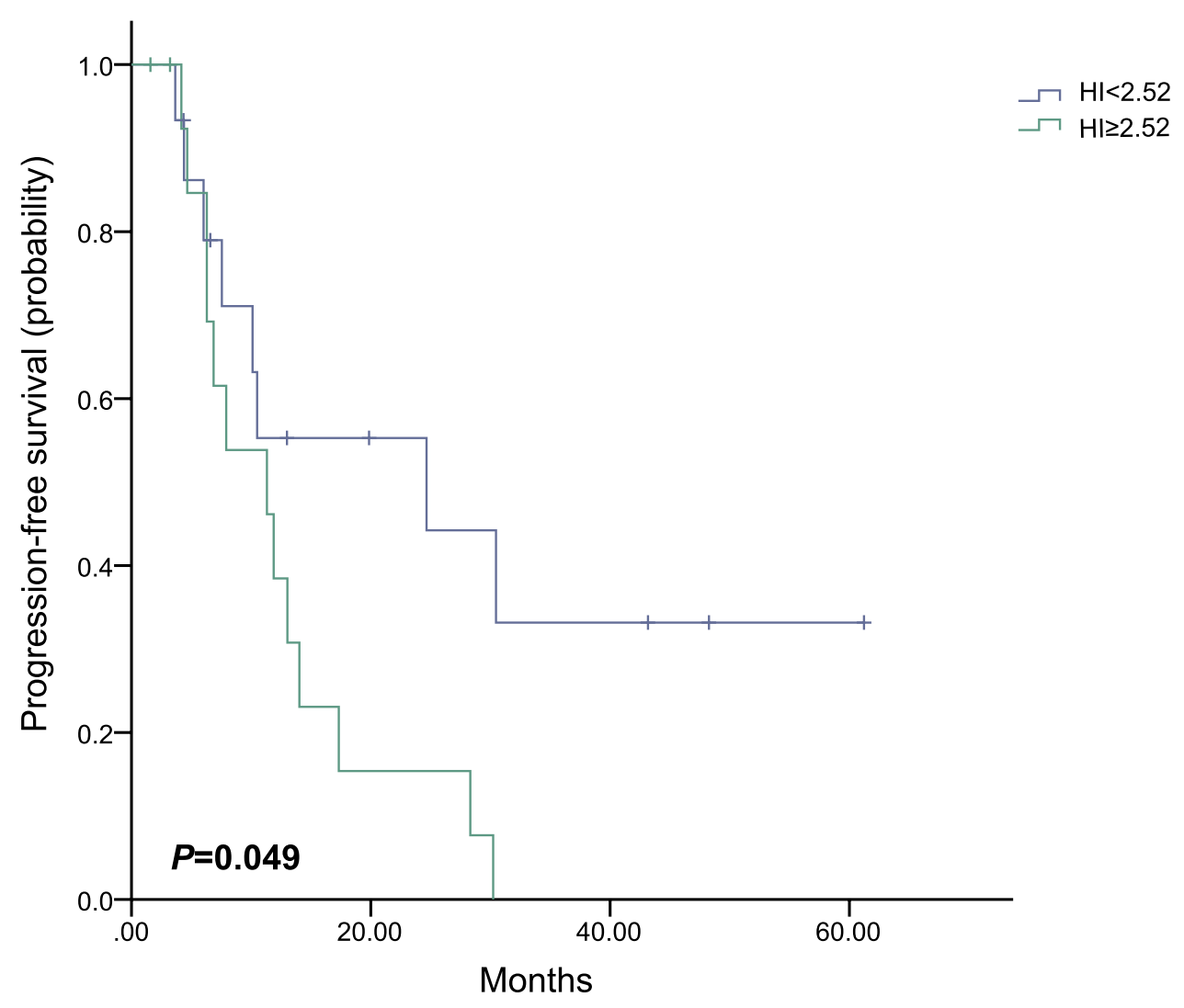

Figure 4 Kaplan-Meier curve of progression-free survival for HER2+ patients stratified by HI.

Abbreviations: HER2, human epidermal growth factor receptor 2; $\mathrm{HI}$, heterogeneity index.

A

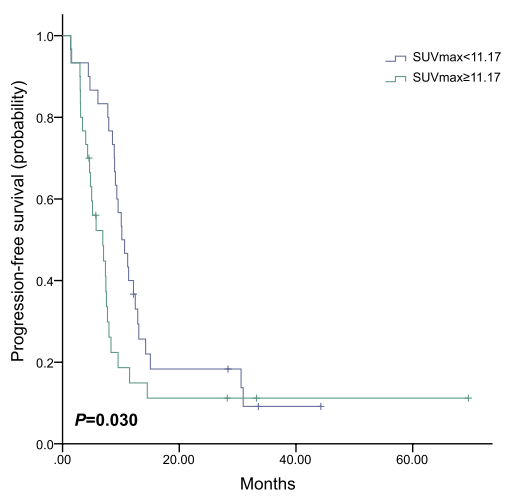

B

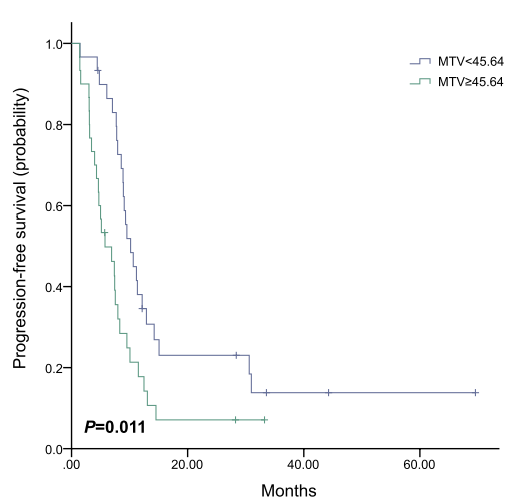

C

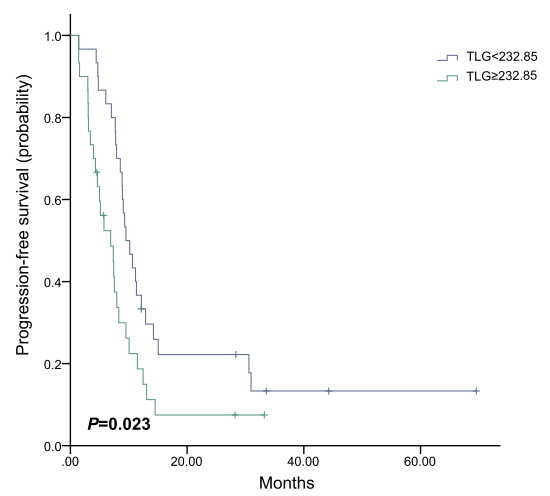

Figure 5 Kaplan-Meier curve of progression-free survival for triple-negative patients stratified by SUVmax (A), MTV (B) and TLG (C). Abbreviations: SUVmax, maximum standardized uptake value; MTV, metabolic tumor volume; TLG, total lesion glycolysis.

TLG or MTV rather than SUVmax was significantly correlated to the OS of MTNBC patients. As explained by the author, the results might be biased due to different PET machines which were likely to have a variety of reconstruction algorithms and were coupled with a large number of small lesions whose SUVmax had inconsistent measured values due to partial volume effects. ${ }^{28}$ SUVmax and HI might be potential predictors for the treatment outcome of mTNBC according to another study which was conducted in our center, ${ }^{29}$ the study only investigated two PET parameters (SUVmax and intratumor HI) and included patients whose first-line treatment was a platinum-containing regimen. ${ }^{29}$ Compared with the 

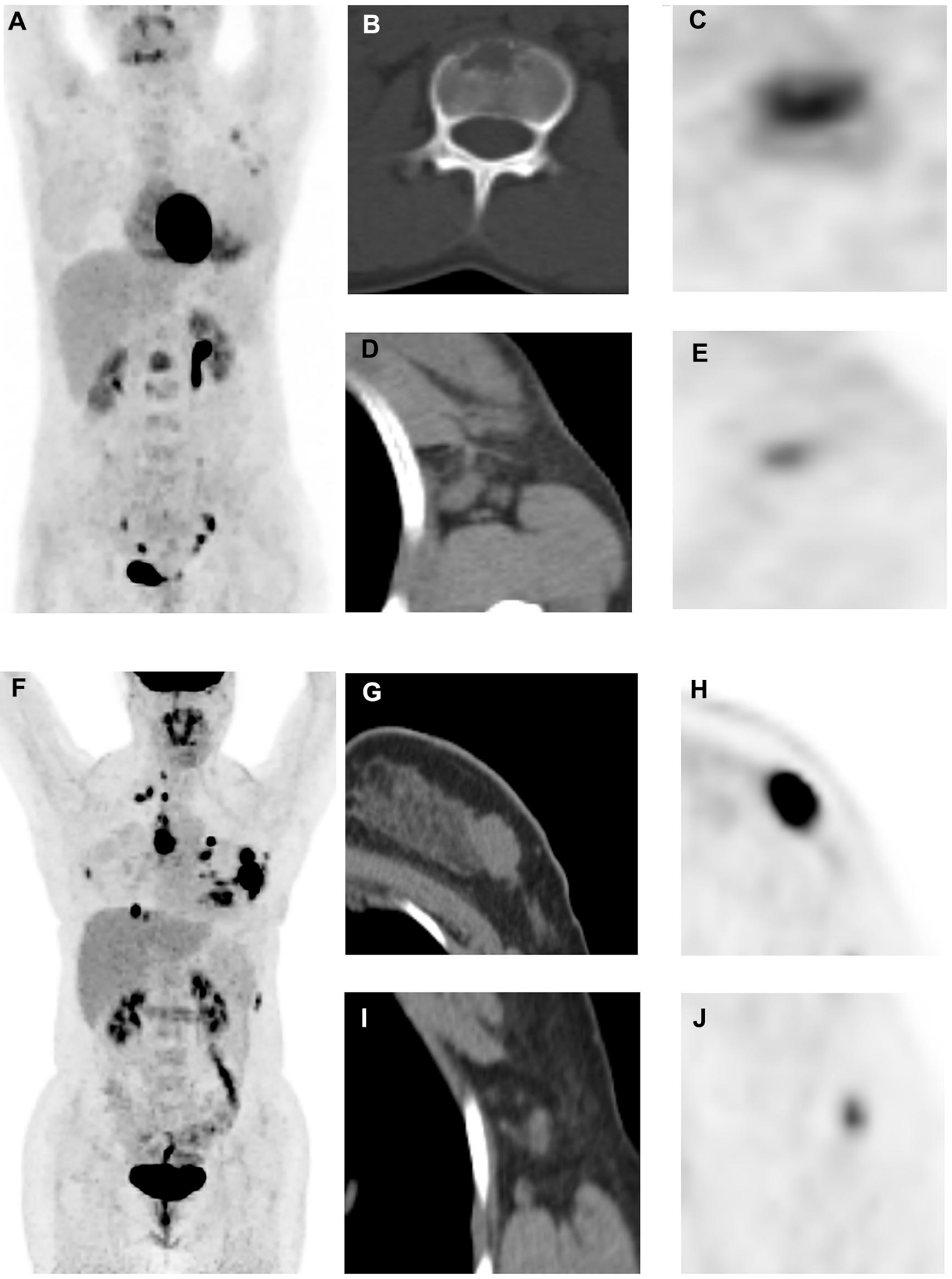

H
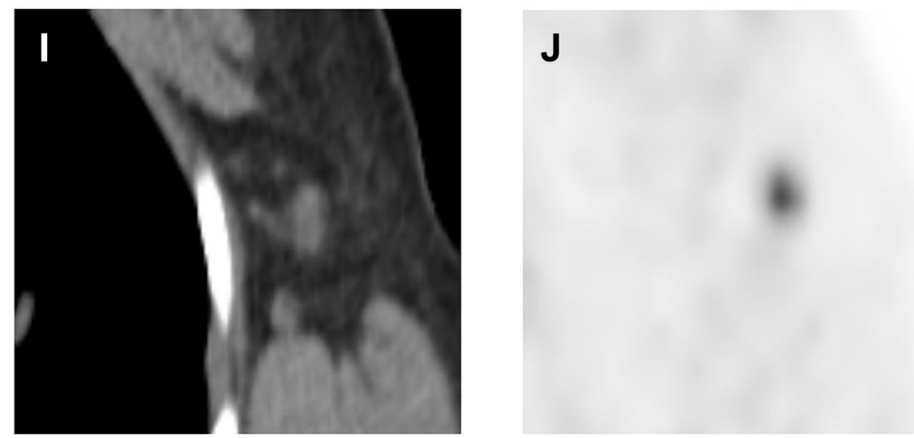

Figure 6 Representative images. (A-E): A 31 -year-old female patient with HR+/HER2- MBC underwent PET/CT scan (A, maximum intensity projection image). We detected that the first lumbar vertebra lesion had the highest I8F-FDG uptake in all metastatic lesions (B, CT image; C, PET image, SUVmax were 5.43), whereas the right axillary lymph node lesion had the lowest uptake (D, CT image; E, PET image, minimum FDG uptake across all lesions = 3.58). Therefore, HI of this patient was I.52, and she had a median PFS of 57.4 months. (F-J): A 30-year-old female patient with HR+/HER2- MBC underwent I8F-FDG PET/CT scan (F, maximum intensity projection image). We detected that the chest wall lesion had the highest I8F-FDG uptake in all metastatic lesions (G, CT image; H, PET image, SUVmax = I5.7I), whereas right axillary lymph node lesion had the lowest uptake (I, CT image; J, PET image, minimum FDG uptake $=3.79)$; Therefore, HI of this patient was 4. I5, and she had a PFS of 5.2 months.

Abbreviations: HR, hormone receptor, HER2, human epidermal growth factor receptor 2; MBC, metastatic breast cancer; PET/CT; positron emission tomography/ computed tomography; I8F-FDG, I8F-fluorodeoxyglucose; SUVmax, maximum standardized uptake value; HI, heterogeneity index, PFS, progression-free survival. 
two studies, however, our study enrolled more patients with $\mathrm{mTNBC}$ and explored more PET parameters using the same PET machine.

Consideration should be given to some limitations of this study. First, retrospective research introduced unavoidably inherent biases. Selection bias might exist in the population. MBC patients in the center might not represent the overall distribution of $\mathrm{MBC}$ because not all of them received PET imaging. Besides, the patients' population was heterogeneous in different molecular subtypes and treatment, which might have an impact on prognosis. Second, more than half of the patients had no metastatic biopsy, and the molecular subtype was determined based on the information of receptor status obtained from the biopsy of primary tumors. Third, cut-off values based on medians have not been validated or used in other studies.

Despite the above limitations, this study explored the predictive value of different PET-derived parameters in metastatic treatment-naive $\mathrm{MBC}$ stratified by different molecular subtypes and De novo stage IV.

\section{Conclusion}

In $\mathrm{MBC}$ patients without receiving first-line treatment, pretreatment PET parameters (SUVmax, SUVmean, MTV, TLG, as well as HI) are statistically significant prognostic variables. However, predictive PET parameters might be different in patients with MBC stratified by De novo stage IV and different molecular subtypes.

\section{Ethical Approval and Consent to Participate}

This study was conducted in accordance with the Declaration of Helsinki. All data were anonymized to comply with the provisions of personal data protection legislation. The Institutional Review Board of Fudan University Shanghai Cancer Center (FUSCC) approved the study (1812195-6). Due to the retrospective nature of this study and due to the fact that only historical medical data were collected, written informed consent was not required.

\section{Author Contributions}

All authors made substantial contributions to conception and design, acquisition of data, or analysis and interpretation of data; took part in drafting the article or revising it critically for important intellectual content; agreed to submit to the current journal; gave final approval for the version to be published; and agreed to be accountable for all aspects of the work.

\section{Funding}

This study was supported by the National Natural Science Foundation of China (Grant No. 81874114 to WBY), the Shanghai Committee of Science and Technology Fund (Grant No.19ZR1411300 to YZY) and by the Chinese Society of Clinical Oncology Youth Committee (CSCO YOUNG). The funding agencies have no role in the actual experimental design, patient recruitment, data collection, analysis, interpretation, or writing of this manuscript.

\section{Disclosure}

The authors report no conflicts of interest in this work.

\section{References}

1. Bray F, Ferlay J, Soerjomataram I, Siegel RL, Torre LA, Jemal A. Global cancer statistics 2018: GLOBOCAN estimates of incidence and mortality worldwide for 36 cancers in 185 countries. CA Cancer J Clin. 2018;68(6):394-424. doi:10.3322/caac. 21492

2. Brewster AM, Hortobagyi GN, Broglio KR, et al. Residual risk of breast cancer recurrence 5 years after adjuvant therapy. $J$ Natl Cancer Inst. 2008;100(16):1179-1183. doi:10.1093/jnci/djn233

3. Howlader NN, Noone AM, Krapcho M et al. SEER cancer statistics review, 1975-2013 (based on November 2015 SEER data submission, posted to the SEER web site, 2016). 2016. Available from: http://seer.cancer.gov/csr/1975_2013/. Accessed May 1, 2021.

4. Deluche E, Antoine A, Bachelot T, et al. Contemporary outcomes of metastatic breast cancer among 22,000 women from the multicentre ESME cohort 2008-2016. Eur $J$ Cancer. 2020;129:60-70. doi:10.1016/j.ejca.2020.01.016

5. Perou CM, Sorlie T, Eisen MB, et al. Molecular portraits of human breast tumours. Nature. 2000;406(6797):747-752. doi:10.1038/ 35021093

6. Kim SJ, Lee SW. Diagnostic accuracy of (18)F-FDG PET/CT for detection of peritoneal carcinomatosis; a systematic review and meta-analysis. Br J Radiol. 2018;91(1081):20170519. doi:10.1259/ bjr.20170519

7. Kadoya T, Aogi K, Kiyoto S, Masumoto N, Sugawara Y, Okada M. Role of maximum standardized uptake value in fluorodeoxyglucose positron emission tomography/computed tomography predicts malignancy grade and prognosis of operable breast cancer: a multi-institute study. Breast Cancer Res Treat. 2013;141(2):269-275. doi:10.1007/ s10549-013-2687-7

8. Aogi K, Kadoya T, Sugawara Y, et al. Utility of (18)F FDG-PET/CT for predicting prognosis of luminal-type breast cancer. Breast Cancer Res Treat. 2015;150(1):209-217. doi:10.1007/s10549-015-3303-9

9. Morris PG, Ulaner GA, Eaton A, et al. Standardized uptake value by positron emission tomography/computed tomography as a prognostic variable in metastatic breast cancer. Cancer-Am Cancer Soc. 2012;118(22):5454-5462.

10. Son SH, Kim DH, Hong CM, et al. Prognostic implication of intratumoral metabolic heterogeneity in invasive ductal carcinoma of the breast. Bmc Cancer. 2014;14:585. doi:10.1186/1471-2407-14-585 
11. Ha S, Park S, Bang JI, Kim EK, Lee HY. Metabolic radiomics for pretreatment (18)F-FDG PET/CT to characterize locally advanced breast cancer: histopathologic characteristics, response to neoadjuvant chemotherapy, and prognosis. Sci Rep. 2017;7(1):1556. doi:10.1038/s41598-017-01524-7

12. Huyge V, Garcia C, Alexiou J, et al. Heterogeneity of metabolic response to systemic therapy in metastatic breast cancer patients. Clin Oncol. 2010;22(10):818-827. doi:10.1016/j.clon.2010.05.021

13. Bural G, Torigian DA, Houseni M, Basu S, Srinivas S, Alavi A. Tumor metabolism measured by partial volume corrected standardized uptake value varies considerably in primary and metastatic sites in patients with lung cancer. A new observation. Hell $\mathrm{J} \mathrm{Nucl} \mathrm{Med}$. 2009;12(3):218-222.

14. Salamon J, Derlin T, Bannas P, et al. Evaluation of intratumoural heterogeneity on (1)(8)F-FDG PET/CT for characterization of peripheral nerve sheath tumours in neurofibromatosis type 1. Eur J Nucl Med Mol Imaging. 2013;40(5):685-692. doi:10.1007/s00259-012-2314-6

15. Tahari AK, Alluri KC, Quon H, Koch W, Wahl RL, Subramaniam RM. FDG PET/CT imaging of oropharyngeal squamous cell carcinoma: characteristics of human papillomavirus-positive and -negative tumors. Clin Nucl Med. 2014;39(3):225-231. doi:10.1097/RLU.0000000000000255

16. Cokmert S, Tanriverdi O, Karapolat I, et al. The maximum standardized uptake value of metastatic site in 18 F-FDG PET/CT predicts molecular subtypes and survival in metastatic breast cancer: an Izmir oncology group study. J Buon. 2016;21(6):1410-1418.

17. Adams MC, Turkington TG, Wilson JM, Wong TZ. A systematic review of the factors affecting accuracy of SUV measurements. AJR Am J Roentgenol. 2010;195(2):310-320. doi:10.2214/AJR.10.4923

18. Larson SM, Erdi Y, Akhurst T, et al. Tumor treatment response based on visual and quantitative changes in global tumor glycolysis using PET-FDG imaging. The visual response score and the change in total lesion glycolysis. Clin Positron Imaging. 1999;2(3):159-171. doi:10.1016/S1095-0397(99)00016-3

19. Ulaner GA, Eaton A, Morris PG, et al. Prognostic value of quantitative fluorodeoxyglucose measurements in newly diagnosed metastatic breast cancer. Cancer Med. 2013;2(5):725-733. doi:10.1002/ cam4.119

20. Fonti R, Larobina M, Del VS, et al. Metabolic tumor volume assessed by $18 \mathrm{~F}-\mathrm{FDG}$ PET/CT for the prediction of outcome in patients with multiple myeloma. $J$ Nucl Med. 2012;53 (12):1829-1835. doi:10.2967/jnumed.112.106500
21. Zhu D, Ma T, Niu Z, et al. Prognostic significance of metabolic parameters measured by (18)F-fluorodeoxyglucose positron emission tomography/computed tomography in patients with small cell lung cancer. Lung Cancer. 2011;73(3):332-337. doi:10.1016/j. lungcan.2011.01.007

22. Yang Z, Shi Q, Zhang Y, et al. Pretreatment (18)F-FDG uptake heterogeneity can predict survival in patients with locally advanced nasopharyngeal carcinoma-a retrospective study. Radiat Oncol. 2015;10:4. doi:10.1186/s13014-014-0268-5

23. Win T, Miles KA, Janes SM, et al. Tumor heterogeneity and permeability as measured on the CT component of PET/CT predict survival in patients with non-small cell lung cancer. Clin Cancer Res. 2013;19 (13):3591-3599. doi:10.1158/1078-0432.CCR-12-1307

24. De Palma M, Hanahan D. The biology of personalized cancer medicine: facing individual complexities underlying hallmark capabilities. Mol Oncol. 2012;6(2):111-127. doi:10.1016/j. molonc.2012.01.011

25. Marusyk A, Polyak K. Tumor heterogeneity: causes and consequences. Biochim Biophys Acta. 2010;1805(1):105-117. doi:10.1016/j.bbcan.2009.11.002

26. Zhang J, Jia Z, Ragaz J, et al. The maximum standardized uptake value of 18 F-FDG PET scan to determine prognosis of hormone-receptor positive metastatic breast cancer. Bmc Cancer. 2013;13:42. doi:10.1186/1471-2407-13-42

27. Jimenez-Ballve A, Garcia GM, Salsidua-Arroyo O, et al. Prognostic value of metabolic tumour volume and total lesion glycolysis in (18) F-FDG PET/CT scans in locally advanced breast cancer staging. Rev Esp Med Nucl Imagen Mol. 2016;35(6):365-372. doi:10.1016/j. remn.2016.01.007

28. Marinelli B, Espinet-Col C, Ulaner GA, et al. Prognostic value of FDG PET/CT-based metabolic tumor volumes in metastatic triple negative breast cancer patients. Am J Nucl Med Mol Imaging. 2016;6(2):120-127.

29. Gong C, Ma G, Hu X, et al. Pretreatment (18)F-FDG uptake heterogeneity predicts treatment outcome of first-line chemotherapy in patients with metastatic triple-negative breast cancer. Oncologist. 2018;23(10):1144-1152. doi:10.1634/theoncologist.2018-0001
International Journal of General Medicine

\section{Publish your work in this journal}

The International Journal of General Medicine is an international, peer-reviewed open-access journal that focuses on general and internal medicine, pathogenesis, epidemiology, diagnosis, monitoring and treatment protocols. The journal is characterized by the rapid reporting of reviews, original research and clinical studies across all disease areas. The manuscript management system is completely online and includes a very quick and fair peer-review system, which is all easy to use. Visit http://www.dovepress.com/ testimonials.php to read real quotes from published authors. 